\title{
An Irish Breast Cancer Survivorship Study: Are We Meeting Our Patients Needs?
}

\section{Abstract :}

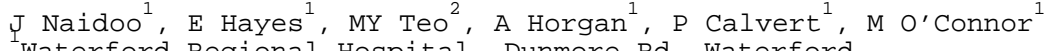
Waterford Regional Hospital, Dunmore Rd, Waterford AMNCH, Tallaght, Dublin 24

\section{Abstract}

Irish breast cancer survivors needs have not been studied. Physical, psychological, social and spiritual concerns were investigated. Patient satisfaction with hospital discharge, GP follow-up, and the benefit of a discharge pack was investigated. A cohort of patients from the South East Cancer Centre was identified. Inclusion criteria: localized breast cancer, completion of adjuvant therapy, GP-led follow-up in the last 5 years. An anonymous questionnaire was developed, and ethical approval obtained. Subgroup analyses for age and time since diagnosis and discharge were completed. 80 patients were identified. 44 patients (55\%) completed the questionnaire, 5 (6\%) were excluded. Commonest completed. 80 patients were identified. 44 patients (55\%) completed the questionnaire, 5 (6\%) were excluded. Commonest concerns included: fatigue (51\%), fear of recurrence (69\%) and second cancers concerns (69\%.) 23 ( $59 \%)$ and 25 patients
$(64 \%)$ were satisfied with discharge and GP follow-up respectively. 27 patients (67\%) reported benefit from a discharge $(64 \%)$ were satisfied with discharge and GP follow-up respectively. 27 patients (67\%) repor
pack. Irish breast cancer survivors had concerns, and were satisfied with GP follow-up.

\section{Introduction}

Breast cancer is the commonest cancer in Ireland, with approximately 2000 cases diagnosed per year. Due to improvements in early detection, screening, diagnosis, and treatment, breast cancer mortality in Ireland has reduced by $24 \%$ in the last five years. Cancer survivorship is a concept pioneered in the $1980 \mathrm{~s}$ by the National Coalition for Cancer Survivorship (NCCS), and refers to the stage of cancer care after completion of active anti-cancer treatment. Clinical care during this period focuses on the encouragement of healthy behaviour, management of complications of therapy, and monitoring for signs of recurrence. In Ireland, this stage of cancer care is managed primarily by hospital-based services. Discharge from these services is not a standardized process across cancer centres. It is thus a challenge for hospital-based specialists to provide ongoing care to the growing number of survivors who have completed treatment. Breast cancer services in Ireland to date have focussed on early detection and treatment to improve patient survival. As the number of breast cancer survivors continues to grow, a model of comprehensive care is needed to address the specialized needs of this cohort of patients.

A number of models of survivor care are in operation in Australia, Canada and the United Kingdom (UK) ${ }^{3-5}$. Survivorship p, yograms in the United States (U.S) and UK divide patients needs into physical, psychological, social and spiritual - Common physical symptoms addressed by survivorship programs include: pain, fatigue, sleep disturbance, nausea/vomiting, reduced appetite, general condition, mobility/balance, ability to perform daily tasks, and hair/skin symptoms. Psychological symptoms include: anger, anxiety, sadness, loss of independence, fear of recurrence, fear of death, depression/low mood, difficulty coping, isolation, future uncertainty, stress, and change ${ }_{7}$ in family roles. Social/supportive concerns refer to financial issues, health insurance, and return to employment. Spiritual concerns include a need for spiritual support, and loss of faith. The core elements of a survivorship plan include: a treatmen summary, follow-up schedule, information regarding short and long-term treatment side effects, signs and symptoms of recurrence, information sent to the GP, lifestyle, nutrition, exercise information and updates to recommended care. Many countries have assessed how and where survivorship care can be best delivered. A Canadian randomized controlled trial demonstrated no difference in health-related quality of life and recurrence-related clinical events, when comparing GP versus hospital-based follow-up for adjuvant breast cancer. A UK study demonstrated that patient satisfaction was better with GP-delivered follow-up cancer care compared to that delivered in hospital departments ${ }^{10}$.

There is no published data on survivor care in Irish patients. Knowledge of their specific survivorship needs and if these needs are currently met, is not known. The primary aim of this study was to assess the needs of an Irish cohort of breast cancer survivors, who had completed care in a hospital-based medical oncology clinic, and were recently discharged to their GP. Needs were stratified into physical, psychological, social/supportive and spiritual. Secondary aims were to assess patient satisfaction with discharge from hospital-based services, satisfaction with GP follow-up, and the possible benefit of introducing a survivorship discharge pack.

\section{Methods}

Patients discharged from a medical oncology specialist clinic of the South East Cancer Centre from 2006-2012, were identified. Inclusion criteria were: a history of localized breast cancer, completion of adjuvant multimodality therapy within the last five years, and discharge from hospital-based oncology services to the GP in the last six years. Patients with metastatic disease, second cancers and male breast cancers were excluded. An anonymous questionnaire was adapted from standardized survivorship questionnaires used in European and American survivorship studies websites of large cancer institutions in the U.S including MD Anderson Cancer centre and the Mayo Clinic ${ }^{14}$. Ethical approval was obtained for the questionnaire from the local ethics committee.

The questionnaire was subdivided into eight subsections. Section one captured patient demographics. Sections two to five dealt with current physical, psychological, supportive/social and spiritual concerns. Sections six and seven questioned patient satisfaction with discharge from the medical oncology clinic. Section eight assessed patients views on methods to improve current survivorship care- such as the supply of a treatment summary and a discharge information pack. Patients graded their concerns as either: no concern, mild, moderate or extreme concern. Subgroup analyses were completed for age, time since discharge and time since diagnosis. Fishers exact test and Cochrane-Armitage tests were used. Patients GPs were contacted to inform them of the questionnaire and its contents, and to confirm patients were still alive. Patients were contacted by the investigators prior to questionnaire postage, to a postarn, to a investigators, the oncology unit, or their GP.

\section{Results}

Eighty patients treated for localized breast cancer were discharged over a six-year period. Forty-four patients (55\%) completed and returned the questionnaire. Five patients were excluded from the study (6\%): 2 patients did not complete the questionnaire in its entirety, 2 patients indicated that they still attended hospital follow-up, and 1 patient had been discharged more than ten years ago. Patient demographic data is summarized in Table 1. 
The most common psychological concern was fear of recurrence, demonstrated in 27 patients (69\%). Psychological concerns are depicted in Figure 2 .

Social/Supportive Care Needs

29 patients (75\%) had no concerns regarding returning to work. 20 patients (52\%) and 22 patients (56\%) did not report any financial and health insurance-related concerns respectively.

Spiritual Needs

Spiritually, the majority of patients had no concerns regarding the need for religious support (84\%) or loss of faith $(89 \%)$.

Secondary aims

Table 2 outlines patients satisfaction with discharge from the specialist clinic. 29 patients (73\%) had been informed of discharge, and 23 (59\%) were satisfied this. 25 patients (64\%) were content with GP cancer-care follow-up. 23 patients (59\%) reported that a treatment summary would have been benefit to them. 59\%, 55\%, 57\% and 34\% of patients felt that they required additional physical, psychological, social and spiritual information respectively. The patients assessment of the services available to them was sought. 21 patients (55\%) did not feel that adequate support group services were available to them. 28 patients (69\%) had concerns regarding second cancers, and 19 patients $(48 \%)$ felt adequate services were lacking to address these concerns.

Statistical Analysis

Patients under 60 years of age were more likely to express physical concerns ( $\mathrm{p}=0.01$.$) Rates of physical and$ psychological concerns decreased with increasing time from diagnosis (p-trend<0.01.) Patients had more psychological concerns in the first two years after discharge to their GPs ( $p=0.017$.

\section{Discussion}

The increasing survival of patients with early stage breast cancers leads to a responsibility for ongoing or survivorship care. Irish patients are monitored by hospital-based services for a number of years after active anti-cancer therapy, and then discharged to their GP for follow-up care. This study aimed to identify the ongoing needs of these patients after discharge from hospital-based services. We identified that the majority of breast cancer survivors have ongoing concerns across a number of domains.

This study demonstrates that a median of 23\% of respondents continue to experience physical problems, $28 \%$ have psychological concerns, 44\% have socialfsupportive issues, and 12\% have spiritual concerns. Similar findings have been reported in larger survivorship studies. A large UK study reported a 50\% incidence of physical symptoms.

Psychologically, cancer survivors have been shown to suffer more distress than those who have never experienced cancer. Interestingly, in this cohort there was a very low incidence of spiritual concerns compared to breast cancer survivors in the U.S. or Europe ${ }^{17}$. This could highlight a unique aspect of Irish survivorship attitudes. In many cases, only mild concerns were expressed. However, the authors acknowledge that the divisions of mild, moderate and extreme concerns can be subjective. Fear of cancer recurrence is known to be one of the chief concerns of cancer survivors 69\% of the study cohort reported this concern. Such concerns are often most effectively addressed by specialists. Thus, the development of specialist-run survivorship programs and national cancer rehabilitation hospitals have been favoured in North America and Nordic countries respectively.

The secondary aims of this study were to assess patient satisfaction with GP-led follow-up care and the provision of a discharge pack. 64\% of respondents were satisfied with GP-led cancer follow-up. Primary care could therefore be considered as a potential model of care for breast cancer survivors in Ireland. Facilitation of direct communication between GPs and specialist oncologists as well as further training and access to services, could improve GP-led cancer care. Survivor care plans have been developed in other countries based on patients needs Medicines 2006 report on Cancer Survivorship and the ASCO Survivorship Task Force, advocates for the supply of a Medicines 206 report on Cancer Survivorship and the Asco Survivorship Task Force, adyocates for the supply of a survivorship care plan to patients on completion of primary anti-cancer therapy and links to survivorship support
follow-up guidelines, information regarding the management of medical concerns, and lis groups. less physical and psychological concerns with increasing time since diagnosis, consistent with improvements in treatment-related toxicity with time. Less psychological concerns were expressed with increasing time since discharge to the GP. A possible explanation could be increased familiarity with GP-led cancer care. 
patients discharged from the same oncology team. It is therefore possible that these patients may not be representative of all Irish breast cancer survivors. Likewise, respondents to the questionnaire could represent those patients with greater concerns. We may therefore be underestimating the concerns of Irish breast cancer survivors overall. A potential solution would be to expand the study to different sites across Ireland. The response rate of the questionnaire is comparable with survey-based studies. However, a greater response rate may be achieved by delivering questionnaires to patients at the time of discharge.

The current model of survivorship care in Ireland faces considerable challenges- increasing numbers of survivors, limited numbers of oncology specialists, multidisciplinary needs, economic constraints, and a disconnect between hospital-based care and community-based resources. This study identifies that Irish breast cancer survivors have ongoing needs after discharge to their GPs. The implementation of a survivorship discharge pack including a treatment summary has the potential to address concerns and improve follow-up care. Most breast cancer survivors in this study favoured GP-led cancer follow-up. Thus we propose that a considerable proportion of survivorship care be delivered by community-based services. Primary care expertise in survivorship care could be supported by the development of a specialist-taught training course aimed at GPs. We hope that the findings of this study will support changes to the implementation and development of a comprehensive Irish survivorship care model.

Correspondence: J Naidoo

Beaumont Hospital, P.O. Box 1297, Beaumont Rd, Dublin 9

Email: jarushka_14@yahoo.com

\section{References}

1. Breast cancer incidence, mortality, treatment and survival in Ireland: 1994-2009. National Cancer Registry, Cork, Ireland (2012). http://www.ncri.ie/pubs/pubfiles/BreastCancer_V12.pdf (Accessed 1 May 2013)

2. Hoffman B, Stovall E. Survivorship perspectives and Advocacy. J Clin Oncol. 2008 Nov; 24: 5154-5159

3. National Health and Medical Research Council. Clinical practice guidelines for the management of early breast cancer. http://www.nhmrc.gov.au/publications/_files/cp74.pdf (Accessed 1 May 2013.)

4. Grunfeld E, Sukhbinder D, Levine M. Clinical practice guidelines for the care and treatment of breast cancer: Follow-up after treatment for breast cancer (2005 update). Can Med Assoc J. May 2005; 172: 1319-1320

5. National Institute of Clinical Excellence: Guidance on Cancer Services. Improving outcomes in breast cancer: Manual update. http://www.nice.org.uk/pdf/Improving_outcomes_breastcancer_manual.pdf (Accessed 1 May 2013)

6. National Coalition for Cancer Survivorship. http://www.canceradvocacy.org (Accessed 1 May 2013)

7. Phillips KM, Jim HS, Small BJ, Laronga C, Andrykowski MA, Jacobsen PB. Cognitive functioning after cancer treatment: a 3-year longitudinal comparison of breast cancer survivors treated with chemotherapy or radiation and non cancer controls. Cancer. 2012 Apr; 118: 1925-32.

8. Earle CC. Failing to plan is planning to fail: improving the quality of care with survivorship care plans. J Clin Oncol. 2006 Nov; 24: 51126

9. Grunfeld E, Levine MN, Julian JA, Coyle D, Szetchtman B, Mirsky D, Verma S, Dent S, Sawka C, Pritchard Kl, Ginsburg $\mathrm{D}$, Wood M, Whelan $\mathrm{T}$. Randomized trial of long-term followup for early-stage breast cancer: comparison of family physician versus specialist care. J Clin Oncol. 2006 Feb; 24: 848-55

10. Grunweld E, Fitzpatrick R, Mant D, Yudkin P, Adewuyi-Dalton R, Stewart J, Cole D, Vessey M. Comparison of breast cancer patient satisfaction with follow-up in primary care versus specialist care: results from a randomized controlled trial. Br J Gen Pract. 1999; 49: 70510

11. Harrison SE, Watson EK, Ward AM, Khan NF, Turner D, Adams E, Forman D, Roche MF, Rose PW. Primary Health and Supportive Care Needs of Long-Term Cancer Survivors: A' Questionnaire Survey. J Clin'Oncol. 2011 Feb; 29: 2091-8

12. Turner A, McHattie D. Adult Cancer Survivorship Programme NHS Birmingham East and North Test Community Case Study Tumour Group: Breast

http://www.birminghamcancer.nhs.uk/uploads/document_file/document/4ce6ce05358e981b58000ccc/care_-_cancer_awareness_and_reco (Accessed 1 May 2013)

13. Ganz PM, Kwan L, Stanton AL, Bower JE, Belin TR. Physical and Psychosocial Recovery in the Year After Primary Treatment of Breast Cancer. J Clin Oncol. 2011 Nov; 21:1101-9

14. Survivorship: Living with, Through and Beyond Cancer:

http://issuu.com/mdandersoncancercenter/docs/survivorship_booklet_final_dec6_2011_(Accessed 1 May 2013)

15. Cancer Survivors Survey of Needs: Pain/Palliative care Resource

http://prc.coh.org/Survivorship/MAYOะ20CSS.pdf (Accessed 1 May 2013)

16. Hoffman KE, McCarthy EP, Recklitis CJ, Ng AK. Psychological distress in long-term survivors of adult-onset cancer: results from a national survey. Arch Intern Med. 2009 Jul; 169:127481.

17. Schlairet M, Heddon M, Griffis M. Piloting a Needs Assessment to Guide Development of Survivorship Program for a Community Cancer Center. Oncology Nursing Forum. 2010 Jul; 37: 501-508

18. Lee-Jones C, Humphris G, Dixon R, Hatcher MB. Fear of cancer recurrencea literature review and proposed cognitive formulation to explain exacerbation of recurrence fears. Psychooncology. 1997 Jun; 6: 95-105

19. Mehta SS, Lubeck DP, Pasta DJ, Litwin MS. Fear of cancer recurrence in patients undergoing definitive treatment for prostate cancer: results from CaPSURE. J Urol. 2003 Nov; 170:1931-1933.

20. Oeffinger KC, MCCabe MS. Models for delivering survivorship care. J Clin Oncol. 2006 Nov 10; 24: 5117-24

21. Nordic Cancer Union. From needs to offers: Rehabilitation of cancer patients. http://www. ncu.nu/patient/rehabilitation.shtmi (Accessed 1 May 2013)

22. Richardson A, Addington-Hall J, Amir Z, Foster C, Stark D, Armes J, Brearley SG, Hodges L, Hook J, Jarret N, Stamataki Z, Scott I, Walker J, Ziegler L, Sharpe M. Knowledge, ignorance and priorities for research in key areas of cancer survivorship: findings from a scoping review. Br J Cancer. 2011 Nov; 105: S82 94

23. Hewitt M, Greenfield S, Stovall E. From Cancer Patient to Cancer Survivor: Lost in Transition. Washington, DC: National Academies Press; 2006

24. Ganz PA. A teachable moment for oncologists: Cancer survivors, 10 million strong and growing! J Clin Oncol. 2005 Aug; 23: 5458-60

25. Kroenke $\mathrm{CH}$, Rosner B, Chen WY, Kawachi I, Colditz GA, Holmes MD. Functional Impact of Breast Cancer by Age at Diagnosis. J Clin Oncol 2004 May; 22: 1849-56 
